\title{
\begin{tabular}{l|l} 
Mibraries & DSpace@MIT
\end{tabular}
}

\author{
MIT Open Access Articles
}

\section{Time-resolved measurements of field-driven domain wall motion in a submicron strip with perpendicular magnetic anisotropy}

The MIT Faculty has made this article openly available. Please share how this access benefits you. Your story matters.

Citation: Emori, Satoru, David C. Bono, and Geoffrey S. D. Beach. Time-resolved Measurements of Field-driven Domain Wall Motion in a Submicron Strip with Perpendicular Magnetic Anisotropy. Journal of Applied Physics 111, no. 7 (2012): 07D304. (C) 2012 American Institute of Physics

As Published: http://dx.doi.org/10.1063/1.3676225

Publisher: American Institute of Physics (AIP)

Persistent URL: http://hdl.handle.net/1721.1/79657

Version: Final published version: final published article, as it appeared in a journal, conference proceedings, or other formally published context

Terms of Use: Article is made available in accordance with the publisher's policy and may be subject to US copyright law. Please refer to the publisher's site for terms of use. 


\section{AIP $\mid$ Applied Physics}

Time-resolved measurements of field-driven domain wall motion in a submicron strip with perpendicular magnetic anisotropy

Satoru Emori, David C. Bono, and Geoffrey S. D. Beach

Citation: J. Appl. Phys. 111, 07D304 (2012); doi: 10.1063/1.3676225

View online: http://dx.doi.org/10.1063/1.3676225

View Table of Contents: http://jap.aip.org/resource/1/JAPIAU/v111/i7

Published by the American Institute of Physics.

\section{Additional information on J. Appl. Phys.}

Journal Homepage: http://jap.aip.org/

Journal Information: http://jap.aip.org/about/about_the_journal

Top downloads: http://jap.aip.org/features/most_downloaded

Information for Authors: http://jap.aip.org/authors

\section{ADVERTISEMENT}

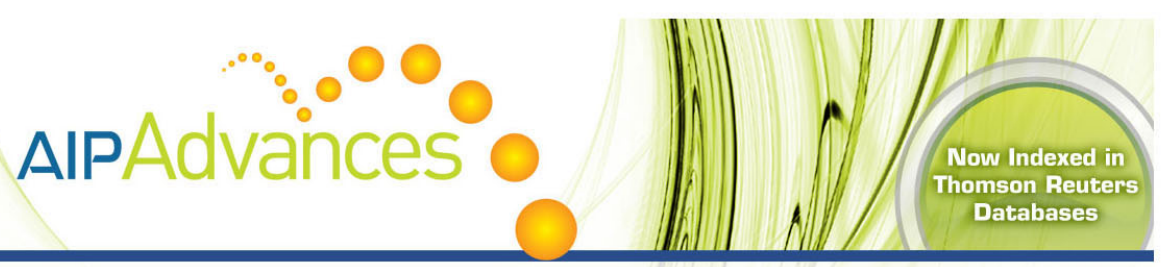

\section{Explore AIP's open access journal: Rapid publication \\ - Article-level metrics \\ - Post-publication rating and commenting}




\title{
Time-resolved measurements of field-driven domain wall motion in a submicron strip with perpendicular magnetic anisotropy
}

\author{
Satoru Emori, ${ }^{\text {a) }}$ David C. Bono, and Geoffrey S. D. Beach \\ Department of Materials Science and Engineering, Massachusetts Institute of Technology, Cambridge, \\ Massachusetts 02139, USA
}

(Presented 1 November 2011; received 23 September 2011; accepted 9 November 2011; published online 5 March 2012)

\begin{abstract}
The motion of domain walls (DWs), driven by magnetic field and spanning almost eight orders of magnitude in velocity, has been studied in a submicron-wide strip of $\mathrm{Co} / \mathrm{Pt}$ with perpendicular magnetic anisotropy. A scanning magneto-optic Kerr effect (MOKE) system is used to conduct time-resolved measurements of DW dynamics in both the thermally activated and viscous flow regimes. MOKE signal transients in the thermally activated regime reveal distributions of stochastic DW propagation events. Transients in the viscous flow regime show deterministic DW motion with velocities $\sim 20 \mathrm{~m} / \mathrm{s}$. The transition between the two dynamic regimes is observed as the relationship between the DW velocity and the driving magnetic field changes from exponential to linear. (C) 2012 American Institute of Physics. [doi:10.1063/1.3676225]
\end{abstract}

\section{INTRODUCTION}

The viability of domain wall (DW)-based spintronic memory ${ }^{1}$ and $\operatorname{logic}^{2}$ devices depends on the understanding and reliable control of DW dynamics. Out-of-plane magnetized submicron strips with perpendicular magnetic anisotropy (PMA), such as those based on $\mathrm{Co} / \mathrm{Pt}$ and $\mathrm{Co} / \mathrm{Ni}$ multilayer thin films, have recently gained much attention for applications to such DW devices. DWs in strips with PMA are about $\sim 10 \mathrm{~nm}$ wide and thus are susceptible to pinning by edge/topographical roughness, grain boundaries, and other nanoscale defects in the strip. ${ }^{3}$ The resulting large pinning fields, typically one or two orders magnitude greater than those of in-plane magnetized NiFe strips, make PMA strip devices robust against stray fields and thermal fluctuations. $\mathrm{DWs}$ in $\mathrm{Co} / \mathrm{Ni}$ devices have also been shown to move by electric current (adiabatic spin transfer torque) independent of the pinning strength. ${ }^{4,5}$

DWs in thin films with PMA exhibit several distinct dynamical regimes due to the large pinning strength, as shown theoretically ${ }^{6}$ and experimentally. ${ }^{7-9}$ Under a small driving force, a DW is subject to a nonuniform pinning energy landscape and therefore moves by the thermally activated process of creep or depinning. In this regime, DW motion is expected to be slow ( $v \ll 10 \mathrm{~m} / \mathrm{s}$ ) and stochastic. Under a sufficiently large driving force, a DW is affected minimally by the pinning landscape and thus flows with a constant mobility $\mu=v / H$, where $v$ is the DW velocity and $H$ is the effective driving field.

DW motion in submicron-wide patterned strips with PMA has been studied with magnetic field, ${ }^{3,10,11}$ electric current, ${ }^{9,12-14}$ and combinations of both ${ }^{4,8,15-17}$ as the driving forces. Many of these studies investigate DW motion in the thermally activated regime with typically a few orders of magnitude in velocity $\left(v \sim 10^{-7}-10^{-3} \mathrm{~m} / \mathrm{s}\right), 3,8,10,11,14-17$

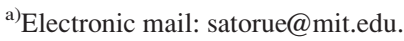

while some others have extracted DW velocities in the viscous flow regime. ${ }^{4,9,12,13}$ However, although the transition between the thermally activated and viscous flow regimes has been observed for continuous thin films with PMA, ${ }^{7-9}$ a study spanning the two regimes for a submicron-wide strip has been lacking. The main challenge for such dynamic characterization is to attain a sufficient temporal resolution to detect DWs moving at $v>10 \mathrm{~m} / \mathrm{s}$, which may be done electrically by monitoring the Hall voltage ${ }^{4,12}$ or magnetoresistance, ${ }^{18,19}$ or optically by measuring the magneto-optic Kerr effect (MOKE). ${ }^{20}$ In this study, a high-bandwidth scanning MOKE system was used to investigate field-driven DW dynamics in an out-of-plane magnetized submicron $\mathrm{Co} / \mathrm{Pt}$ multilayer strip. From these time-resolved measurements, we have characterized DW dynamics spanning nearly eight decades in velocity, identifying the transition between the thermally activated and viscous flow regimes and the corresponding transition from stochastic to deterministic DW motion.

\section{EXPERIMENT}

A $550 \mathrm{~nm}$ wide $\mathrm{Co} / \mathrm{Pt}$ strip was fabricated with e-beam lithography, sputtering, and lift-off. The $\mathrm{Co} / \mathrm{Pt}$ multilayer film had a structure $\mathrm{SiO}_{2}(50) / \mathrm{TaOx}(4) / \mathrm{Pt}(1.6) /[\mathrm{Co}(0.7) /$ $\mathrm{Pt}(1.0)]_{2} / \mathrm{Co}(0.7) / \mathrm{Pt}(1.6)$ (thicknesses in $\mathrm{nm}$ ), similar to those fabricated in Refs. 17 and 21. The magnetic hysteresis loop of the strip is square as shown in Fig. 1(a), indicating the presence of strong PMA and magnetization reversal governed by DW nucleation. $\mathrm{Cu}$ electrodes (100 nm thick) with a Ta adhesion layer were overlaid using a second set of lithographic steps. The scanning electron micrograph in Fig. 1(b) shows the strip and electrodes.

DW motion at room temperature was detected using a scanning MOKE system with a stage motion resolution of $50 \mathrm{~nm}$. A laser beam $(\lambda=532 \mathrm{~nm})$ with an attenuated incident power of $1 \mathrm{~mW}$ was focused to a $\sim 2 \mu \mathrm{m}$ spot size. The 
(a)

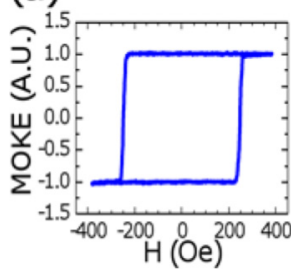

(b)

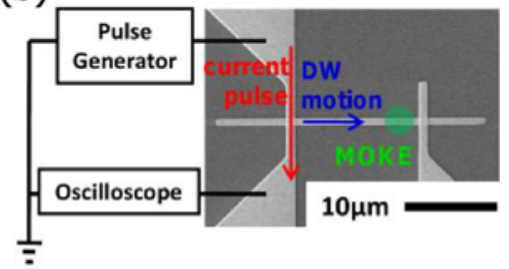

FIG. 1. (Color online) (a) MOKE hysteresis loop obtained at the center of the Co/Pt strip with magnetic field sweep rate $\sim 20 \mathrm{kOe} / \mathrm{s}$ and 100 cycles averaged. (b) Scanning electron micrograph of the Co/Pt strip and electrodes, along with a schematic of the DW nucleation and detection.

MOKE signal was detected with a photomultiplier tube (PMT) with a rise time of $\sim 10 \mathrm{~ns}$. The measurement cycle was similar to that in Ref. 17. A DW was nucleated in the initially saturated $\mathrm{Co} / \mathrm{Pt}$ strip using a local Oersted field from $\mathrm{a} \sim 25 \mathrm{~ns}, 180 \mathrm{~mA}$ current pulse through the $\mathrm{Cu}$ line orthogonal to the strip [Fig. 1(b)]. A driving field from a perpendicular electromagnet subsequently propagated the DW, which was detected with the focused laser spot (MOKE) placed at a known position on the strip. Slower DW motion with arrival times $>1 \mathrm{~ms}$ was detected using a DC-coupled channel from the PMT, connected to a $10-30 \mathrm{kHz}$ bandpass filter, allowing for a signal-to-noise ratio of $>10$ in single-shot measurements. The MOKE signal for faster DW motion was measured through an AC-coupled channel and a digital scope with a 1-GHz sample acquisition rate, permitting the detection of DW motion with a resolution of $\sim 10 \mathrm{~ns}$.

\section{RESULTS AND DISCUSSION}

The stochasticity of thermally activated DW motion in the low drive-field regime is illustrated in Fig. 2. Single-shot MOKE signal transients measured at the same position and driving field [Fig. 2(a)] show magnetization switching due to DW motion at different times, some with intermediate steps corresponding to pinning within the probed region. This stochastic DW motion is manifested in a distribution of switch-

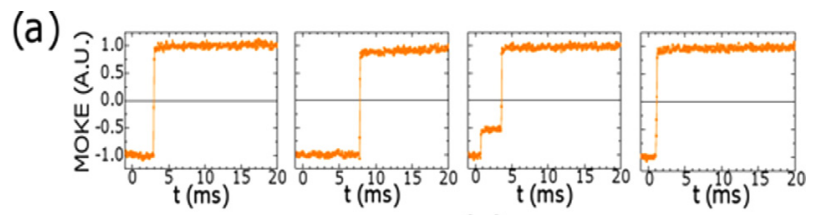

(b)

(c)
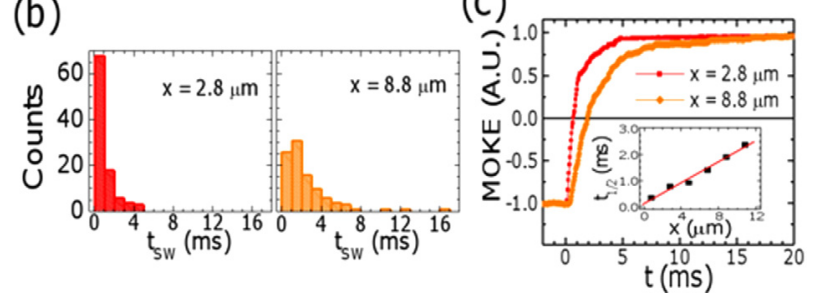

FIG. 2. (Color online) Stochastic nature of thermally activated DW motion, measured at driving field $H=152$ Oe. (a) Single-shot MOKE signal transients measured at $x=8.8 \mu \mathrm{m}$. (b) Distribution of switching times $t_{s w}$ due to DW motion 2.8 and $8.8 \mu \mathrm{m}$ away from the point of DW nucleation. (c) Averaged DW transients at different points on the strip (each an average of 100 transients). The inset shows a plot of average DW arrival time $t_{1 / 2}$ against probed position, with the linear fit used to estimate the DW velocity $v=4.9 \times 10^{-3} \mathrm{~m} / \mathrm{s}$.

ing times $t_{s w}$, defined as the time at which the normalized MOKE signal switches sign. The histograms at two different probed positions on the strip [Fig. 2(b)] show that the distribution of $t_{s w}$ is broader and skewed toward longer times farther away from the point of DW nucleation. Averaged curves of 100 MOKE transients [Fig. 2(c)] become exponential-decay curves, again showing greater breadth with increasing distance from the point of DW nucleation. The average DW arrival time $t_{1 / 2}$ is defined as the time at which the probability of switching due to DW motion is $50 \%$, i.e., half life of the exponential decay. Unless otherwise indicated, averaged MOKE transients were measured $x=8.8 \mu \mathrm{m}$ away from the point of DW nucleation, so that the average DW velocity was calculated as $v=x / t_{1 / 2}$.

For faster DW motion, with arrival times approaching the time scale of the $\sim 25$ ns nucleation pulse, the MOKE signal was measured at six different positions along the strip. To gain a sufficient signal-to-noise ratio on the high bandwidth channel, the MOKE signal was averaged over 500 cycles. Averaged transients at driving fields $H<220$ Oe as illustrated in Fig. 3(a) are fitted well with exponential decay curves, with greater temporal spread in transient signals farther away from the point of DW nucleation. As shown previously by the low-bandwidth measurements, the exponential decay profiles exhibiting greater breadth with increasing distance from the nucleation point emerge from a statistical distribution in DW arrival times, indicating that DW motion is stochastic and thermally activated in this field regime. The DW arrival time $t_{1 / 2}$ is plotted against probed position, and the DW velocity was extracted by linear regression, as shown in Fig. 3(c).

At higher driving fields $H>220$ Oe, the averaged MOKE transients [Fig. 3(b)] are better fitted with error function curves. The temporal width of the averaged reversal

(a)

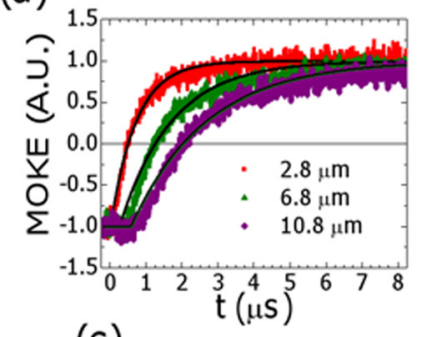

(c)

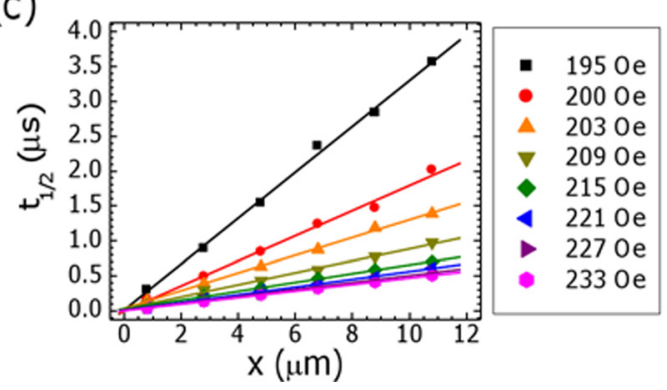

FIG. 3. (Color online) Averaged reversal transients at driving fields (a) $H=200$ Oe $(v=5.6 \mathrm{~m} / \mathrm{s})$ and $(\mathrm{b}) H=233 \mathrm{Oe}(v=21.6 \mathrm{~m} / \mathrm{s})$, measured at different positions away from the point of DW nucleation. The continuous curves show (a) exponential decay and (b) error function fits to the data. (b) Plot of average DW arrival time $t_{1 / 2}$ as a function of probed position. DW velocities are extracted from linear fits. 

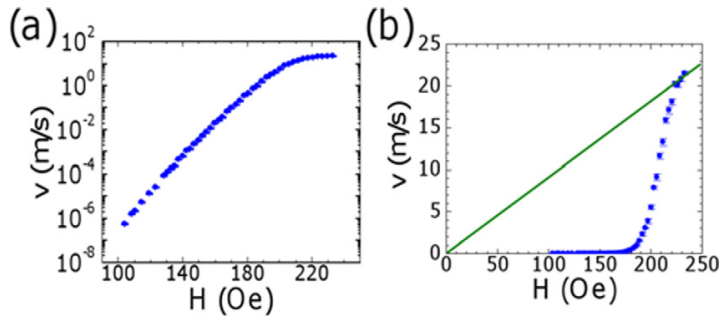

FIG. 4. (Color online) (a) Logarithm of DW velocity $v$ plotted against driving field $H$. (b) $v$ plotted against $H$, with the line serving as a guide for the eye to estimate the DW mobility $\mu \sim 0.09 \mathrm{~m} /(\mathrm{s} \mathrm{Oe})$.

transients, on the order of $100 \mathrm{~ns}$, is independent of the probed position, indicating that DWs passed through the probed regions deterministically. The velocity is again extracted by linearly fitting the DW arrival time $t_{1 / 2}$ (halfway time of the error function curve) versus probed position [Fig. $3(\mathrm{c})]$. At the highest driving field shown, ${ }^{22}$ the DW velocity exceeds $20 \mathrm{~m} / \mathrm{s}$. The ratio of the laser spot size to the temporal width of the reversal transients [Fig. 3(b)] roughly corresponds to this extracted velocity, implying that the width of the reversal transients resulted from DW transit time through the Gaussian spot profile, rather than the statistical distribution in arrival times due to stochastic motion. As DWs move rapidly and deterministically, these data indicate that DW dynamics in this driving field range are in the viscous flow regime.

In Fig. 4, we lastly present the measured velocity-field characteristic for DW motion in this $\mathrm{Co} / \mathrm{Pt}$ strip for a field range spanning 104-233 Oe, corresponding to nearly eight decades in DW velocity. Up to $H \sim 220 \mathrm{Oe}$, the DW velocity increases exponentially with the driving field. Since thermally activated dynamics follow an Arrhenius-type relation $v \sim \exp [-E(H) / k T]$, where $E(H)$ is the activation energy barrier determined by the driving field, the exponential increase in velocity is another indication that DW motion is thermally activated at $H<220$ Oe. Above $H>220$ Oe, the DW velocity scales much more weakly with field, approaching a linear $v$-versus- $H$ characteristic. DW motion above this critical field is therefore governed by viscous flow dynamics, consistent with the MOKE transient measurements. From these data, we estimate a DW mobility $(\mu=v / H)$ of $0.09 \mathrm{~m} /(\mathrm{s}$ Oe), comparable to the mobility extracted from domain expansion measurements in a continuous $\mathrm{Pt} / \mathrm{Co} / \mathrm{Pt}$ thin film, $\sim 0.04 \mathrm{~m} /(\mathrm{s} \mathrm{Oe})$, reported in Ref. 7 .

\section{CONCLUSION}

We have demonstrated the use of a scanning, timeresolved MOKE technique to characterize a wide range of DW dynamics in an out-of-plane magnetized submicron $\mathrm{Co} /$ Pt strip. Low bandwidth measurements show a distribution of magnetization switching times from stochastic, thermally activated DW motion. High bandwidth measurements reveal distinct regimes of DW dynamics corresponding to thermally activated and viscous flow dynamics, along with a maximum DW velocity exceeding $20 \mathrm{~m} / \mathrm{s}$. The velocity-field characteristic for field-driven DW motion, spanning almost eight orders of magnitude, scales exponentially in the thermally activated regime and linearly in the viscous flow regime. Although this study has used only magnetic field as the driving force, this MOKE technique can be extended to investigate the effect on DW dynamics from other driving forces, such as spin transfer torque and current-induced fields.

\section{ACKNOWLEDGMENTS}

Facilities in the MIT RLE Nanostructures Laboratory and the MIT Center for Materials Science and Engineering were used. S.E. was supported by the NSF Graduate Research Fellowship.

${ }^{1}$ S. S. P. Parkin, M. Hayashi, and L. Thomas, Science 320, 190 (2008).

${ }^{2}$ D. A. Allwood, G. Xiong, C. C. Faulkner, D. Atkinson, D. Petit, and R. P. Cowburn, Science 309, 1688 (2005).

${ }^{3}$ F. Cayssol, D. Ravelosona, C. Chappert, J. Ferré, and J. P. Jamet, Phys. Rev. Lett. 92, 107202 (2004).

${ }^{4}$ T. Koyama, D. Chiba, K. Ueda, H. Tanigawa, S. Fukami, T. Suzuki, N. Ohshima, N. Ishiwata, Y. Nakatani, and T. Ono, Appl. Phys. Lett. 98, 192509 (2011).

${ }^{5}$ S. Fukami, T. Suzuki, K. Nagahara, N. Ohshima, and N. Ishiwata, J. Appl. Phys. 108, 113914 (2010).

${ }^{6}$ M. Müller, D. A. Gorokhov, and G. Blatter, Phys. Rev. B 63, 184305 (2001).

${ }^{7}$ P. J. Metaxas, J. P. Jamet, A. Mougin, M. Cormier, J. Ferré, V. Baltz, B. Rodmacq, B. Dieny, and R. L. Stamps, Phys. Rev. Lett. 99, 217208 (2007).

${ }^{8}$ M. Cormier, A. Mougin, J. Ferré, J.-P. Jamet, R. Weil, J. Fassbender, V. Baltz, and B. Rodmacq, J. Phys. D: Appl. Phys. 44, 215002 (2011).

${ }^{9}$ I. M. Miron, T. Moore, H. Szambolics, L. D. Buda-Prejbeanu, S. Auffret, B. Rodmacq, S. Pizzini, J. Vogel, M. Bonfim, A. Schuhl, and G. Gaudin, Nature Mater. 10, 419 (2011).

${ }^{10}$ K.-J. Kim, J.-C. Lee, S.-M. Ahn, K.-S. Lee, C.-W. Lee, Y. J. Cho, S. Seo, K.-H. Shin, S.-B. Choe, and H.-W. Lee, Nature 458, 740 (2009).

${ }^{11}$ R. Lavrijsen, M. A. Verheijen, B. Barcones, J. T. Kohlhepp, H. J. M. Swagten, and B. Koopmans, Appl. Phys. Lett. 98, 132502 (2011).

${ }^{12}$ D. Chiba, G. Yamada, T. Koyama, K. Ueda, H. Tanigawa, S. Fukami, T. Suzuki, N. Ohshima, N. Ishiwata, Y. Nakatani, and T. Ono, Appl. Phys. Express 3, 073004 (2010).

${ }^{13}$ T. A. Moore, I. M. Miron, G. Gaudin, G. Serret, S. Auffret, B. Rodmacq, A. Schuhl, S. Pizzini, J. Vogel, and M. Bonfim, Appl. Phys. Lett. 93, 262504 (2008).

${ }^{14}$ K.-J. Kim, J.-C. Lee, S.-J. Yun, G.-H. Gim, K.-S. Lee, S.-B. Choe, and K.-H. Shin, Appl. Phys. Express 3, 083001 (2010).

${ }^{15}$ L. San Emeterio Alvarez, K.-Y. Wang, S. Lepadatu, S. Landi, S. J. Bending, and C. H. Marrows, Phys. Rev. Lett. 104, 137205 (2010).

${ }^{16}$ J.-C. Lee, K.-J. Kim, J. Ryu, K.-W. Moon, S.-J. Yun, G.-H. Gim, K.-S. Lee, K.-H. Shin, H.-W. Lee, and S.-B. Choe, Phys. Rev. Lett. 107, 067201 (2011).

${ }^{17}$ S. Emori and G. Beach, J. Phys.: Condens. Matter 24, 024214 (2012).

${ }^{18}$ M. Hayashi, L. Thomas, Y. B. Bazaliy, C. Rettner, R. Moriya, X. Jiang, and S. S. P. Parkin, Phys. Rev. Lett. 96, 197207 (2006).

${ }^{19}$ X. Jiang, L. Thomas, R. Moriya, M. Hayashi, B. Bergman, C. Rettner, and S. S. P. Parkin, Nat. Commun. 1, 25 (2010).

${ }^{20}$ G. S. D. Beach, C. Nistor, C. Knutson, M. Tsoi, and J. L. Erskine, Nature Mater. 4, 741 (2005).

${ }^{21}$ S. Emori and G. S. D. Beach, J. Appl. Phys. 110, 033919 (2011).

${ }^{22}$ At even higher fields $H>235 \mathrm{Oe}$, spontaneous nucleation of DWs became prevalent, and it was not possible to correlate DW transit time with respect to probed position. 